\title{
Altered short term heart rate variability with spinal cord stimulation in chronic refractory angina: evidence for the presence of procedure related cardiac sympathetic blockade
}

\author{
R Moore, D Groves, J Nolan, D Scutt, J Pumprla, M R Chester
}

Heart 2004;90:211-212. doi: 10.1136/hrt.2002.002998

$\mathrm{T}$ he first spinal cord stimulator was implanted for intractable angina in 1987. Following this innovation there followed extensive scrutiny of neurostimulation in patients suffering from coronary artery disease, with several observational trials demonstrating the ability of spinal cord stimulation (SCS) to diminish angina, reduce the frequency of hospital admissions, and improve quality of life.

SCS moderates the symptoms of angina by inhibition of pain perception through the release of spinal inhibitory neurotransmitters at the level of the dorsal horn, and also potentially by liberation of cardiac $\beta$ endorphins. In addition it has been established that neurostimulation also achieves its benefit in part by reduction in cardiac ischaemia. ${ }^{1}$ The proposed mechanism for this observation is altered cardiac autonomic balance.

Heart rate variability (HRV) has been used to verify this proposed influence on cardiac autonomic tone. The two trials published to date, however, have failed to demonstrate any such effect. ${ }^{3}$ Both studies used long term Holter ( 24 and 48 hour) ECG recording intervals in which baseline conditions were not controlled and spinal cord stimulators were activated only for a minority of the post-implant recording periods. The aim of this study was to use short term HRV recordings to assess directly the influence of SCS on HRV in a controlled experimental setting.

\section{METHODS}

Sixteen consecutive patients with chronic refractory angina that was successfully controlled with SCS were recruited from the neurostimulation clinic at the National Refractory Angina Centre, The Cardiothoracic Centre, Liverpool, from May to July 2001.

All patients had a Medtronic Pisces quad plus electrode and a Medtronic XTREL II pulse generator implanted. Two patients were ineligible for the study on the basis of multiple atrial and ventricular ectopic beats, which prevented assessment of short term HRV.

Each patient was isolated for a period of 30 minutes before HRV recording. A five minute high resolution $1000 \mathrm{~Hz} \mathrm{HRV}$ recording was taken with the SCS set at zero output, maximum comfortable output and half maximum output with the pulse width, frequency, and electrode settings left unaltered from their usual treatment positions. To investigate whether the order of recording was capable of biasing results, seven patients underwent repeat recording without altering the SCS output from its original settings for the three sequential five minute measurements. All the recordings were made between 2-4 pm in a secluded room with an ambient temperature maintained at approximately $21^{\circ} \mathrm{C}$ and the patient in a seated position.
The data were acquired and processed using the VariaCardio TF4 HRV monitor, which complies with the recommendations of the joint American and European task force. ${ }^{4}$ All datasets were checked for ectopic beats/noise and only datasets, which required three RR intervals or less to be deleted, were included in the analysis. The power spectrum density was calculated using an established coarse grain fast Fourier transformation method.

Differences between the HRV parameters at each SCS output setting were evaluated using the paired Student's $t$ test. Variables with skewed distribution were normalised using logarithmic transformation ( $\log _{\mathrm{e}}$ ) before analysis. A probability value of $\mathrm{p}<0.05$ was considered significant. Values are expressed as mean (SD).

\section{RESULTS}

A total of 14 male patients, aged 40-77 years, were investigated. All patients had been previously diagnosed as suffering from refractory angina, and the majority of study participants (93\%) had previously undergone either coronary artery bypass surgery or percutaneous coronary angioplasty.

The mean low frequency/high frequency (LF/HF) ratio with full power SCS was significantly reduced when compared to recordings taken during no stimulation $(\mathrm{p}=0.024)$ (table 1). The mean LF/HF ratio was reduced, although not significantly, with stimulation at half usual output when compared to baseline $(\mathrm{p}=0.498)$.

The mean natural logarithm of low frequency ( $\log _{\mathrm{e}} \mathrm{LF}$ ) and mean normalised LF power were both significantly lower with no stimulation than with full power stimulation ( $\mathrm{p}=0.001$ and $\mathrm{p}=0.01$, respectively) and with half power stimulation $(\mathrm{p}=0.001$ and $\mathrm{p}=0.01$, respectively).

There was no significant difference comparing full power to half power SCS $(\mathrm{p}=0.493, \mathrm{p}=0.939)$.

There were no significant differences in absolute HF power with SCS calibrated at the three different output settings. There was no significant alteration in heart rate, or arterial blood pressure at the three SCS settings. No patient reported angina during the study.

Seven patients underwent three, five minute recordings without altering the SCS settings (full output). In this group there was no significant difference between any of the HRV parameters.

\section{DISCUSSION}

SCS in this study significantly altered spectral power parameters in HRV. The LF/HF ratio decreased significantly

Abbreviations: HF, high frequency; HRV, heart rate variability; LF, low frequency; SCS, spinal cord stimulation 
Table 1 Mean spectral power at different SCS settings

\begin{tabular}{|c|c|c|c|c|c|c|c|c|c|c|c|c|c|c|c|}
\hline \multirow{4}{*}{$\begin{array}{l}\text { Recording } \\
\text { Mean } \\
\text { SD }\end{array}$} & \multicolumn{3}{|c|}{ LF/HF ratio } & \multicolumn{3}{|l|}{ LF } & \multicolumn{3}{|l|}{ HF } & \multicolumn{3}{|c|}{ Normalised LF } & \multicolumn{3}{|c|}{ Normalised HF } \\
\hline & Off & Half & Full & Off & Half & Full & Off & Half & Full & Off & Half & Full & Off & Half & Full \\
\hline & 1.59 & 1.41 & 0.83 & 381 & 188 & 209 & 400 & 542 & 458 & 0.47 & 0.34 & 0.35 & 0.53 & 0.66 & 0.65 \\
\hline & 2.00 & 2.46 & 1.08 & 688 & 298 & 369 & 677 & 1091 & 879 & 0.25 & 0.26 & 0.21 & 0.25 & 0.26 & 0.21 \\
\hline
\end{tabular}

$H F$, high frequency; LF, low frequency.

with spinal stimulation, as did absolute LF and normalised LF spectral power density. These observations are consistent with reduced cardiac sympathetic activity during SCS.

Researchers have previously demonstrated indirect evidence of reduced cardiac sympathetic activity with SCS in man, and in a recent publication Foreman and colleagues ${ }^{5}$ reported a suppressive effect of SCS on intrinsic cardiac sympathetic activity in dogs undergoing coronary artery ligature. SCS is maximised at the lower cervical and upper thoracic spinal segments, which correspond directly with the portion of the cord responsible for sympathetic outflow. Investigation with SCS has revealed a generalised local "field effect" with reduced neural activity mediated by alteration in the balance of inhibitory and excitatory neurotransmitters. Therefore, although SCS could alter parasympathetic activity both directly and through interspinal neurons, its dominant influence would be anticipated to be directly through diminution of spinal sympathetic activity.

This study also demonstrated that LF spectral components were significantly diminished in the absence of perceived paraesthesia with SCS. It may be postulated that prolonged stimulation at this lower level could offer lessening in the total ischaemic burden and possibly altered arrhythmia thresholds for this patient group.

The order of SCS settings was not randomised, which could provide a confounding bias. The study was only partially blinded, as patients were aware of neurostimulation at full output. In addition the changes in cardiac autonomic balance observed with SCS in this study were evident immediately after switching between output settings. Further work is required to see if these effects are more persistent with increasing periods of stimulation.

SCS is becoming more and more widely used to treat patients suffering with chronic refractory angina. This study shows for the first time that stimulation of the spinal cord interferes with cardiac autonomic nerve traffic and suggests a possible mechanism of action through reduced resting cardiac sympathetic tone. Further clinical studies into the effect of low output stimulation on angina and ischaemia are warranted.

\section{Authors' affiliations}

R Moore, D Groves, M R Chester, National Refractory Angina Centre, CTC, Liverpool, UK

J Nolan, Cardiothoracic Centre, North Staffordshire Hospital, Stoke on Trent, UK

D Scutt, Department of Medical Imaging, Faculty of Medicine, University of Liverpool, Liverpool, UK

J Pumprla, Research Group Functional Rehabilitation and Group Education, Institute of Biomedical Engineering and Physics, University of Vienna, Austria

Correspondence to: Dr Roger Moore, The National Refractory Angina Centre, The Cardiothoracic Centre Liverpool NHS Trust, Thomas Drive, Liverpool, L14 3PE, UK; alison.ithnin@ctc.nhs.uk

Accepted 19 June 2003

\section{REFERENCES}

1 Dejongste $M$, Haaksma J, Hautvast R, et al. Effects of spinal cord stimulation on myocardial ischaemia during daily life in patients with severe coronary artery disease. A prospective ambulatory electrocardiographic study. $\mathrm{Br}$ Heart J 1994;71:413-8

2 Anderson C. Does heart rate variability change in angina pectoris patients treated with spinal cord stimulation? Cardiology 1998;89:14-18.

3 Hautvast R, Brouwer J, Dejongste $M$, et al. Effect of spinal cord stimulation on heart rate variability and myocardial ischaemia in patients with intractable angina - a prospective ambulatory electrocardiographic study. Clin Cardiol 1998:21:33-8.

4 Task Force of the European Society of Cardiology and the North American Society of Pacing and Electrophysiology. Heart rate variability: standards of measurement, physiological interpretation and clinical use. Circulation 1996:93:1043-65.

5 Foreman RD, Linderoth B, Ardell J, et al. Modulation of intrinsic cardiac neurons by spinal cord stimulation: implications for its therapeutic use in angina pectoris. Cardiovasc Res 2000;47:367-75.

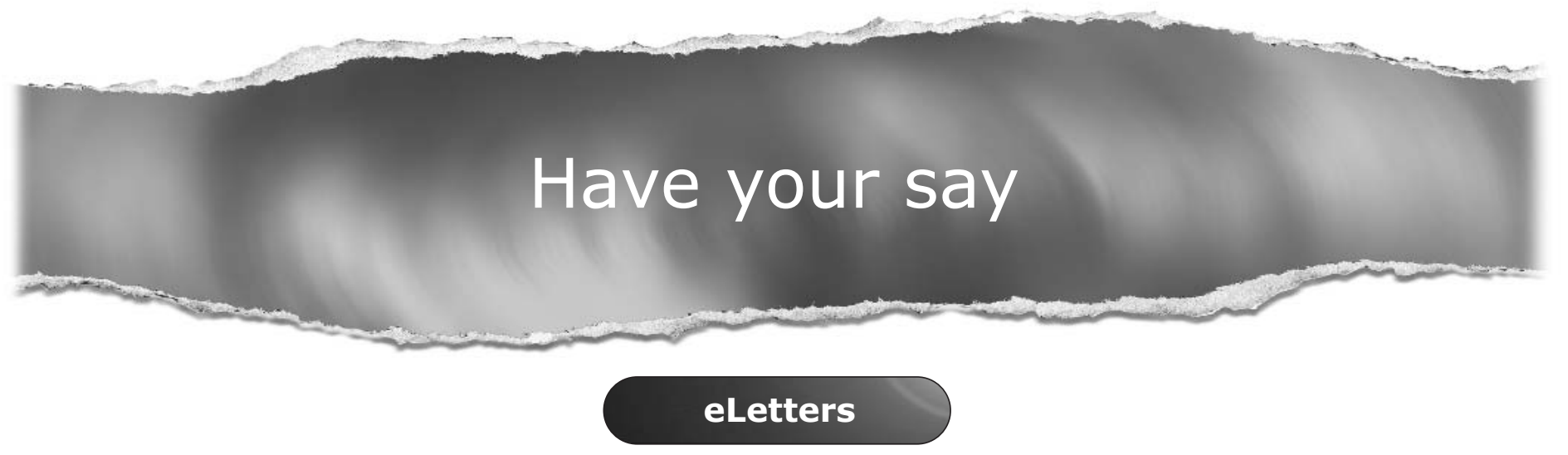

If you wish to comment on any article published in Heart you can send an eLetter using the eletters link at the beginning of each article. Your response will be posted on Heart online within a few days of receipt (subject to editorial screening). 